\title{
ORIGINAL
}

\section{ANÁLISIS COSTE-EFECTIVIDAD DE LA ADHESIÓN INICIAL A LA TERAPIA ANTIRRETROVIRAL ENTRE INDIVIDUOS INFECTADOS POR EL VIH EN BELO HORIZONTE, BRASIL (*)}

\author{
Francisco de Assis Acurcio (1,2), Jaume Puig-Junoy (3), Palmira de Fátima Bonolo (2,4), Maria \\ das Graças Braga Ceccato (2) y Mark Drew Crosland Guimarães (2) \\ (1) Universidade Federal de Minas Gerais. Departamento de Farmácia Social. \\ (2) Universidade Federal de Minas Gerais. Departamento de Medicina Preventiva e Social \\ (3) Universitat Pompeu Fabra. Centro de Investigación en Economía y Salud (CRES). Departamento de Economia y \\ Empresa. \\ (4) Secretaria Municipal de Saúde de Belo Horizonte \\ Beca: CAPES - Coordenação de Aperfeiçoamento de Pessoal de Nível Superior, Brasil. (Programa Post-Doctoral de \\ Francisco de Assis Acurcio en el Centro de Investigación en Economía y Salud, Universitat Pompeu Fabra).
}

\section{RESUMEN}

Fundamento: En Brasil, los antirretrovirales (ARV) se distribuyen a los portadores de VIH de forma gratuita por el Sistema Único de Salud. La adhesión al tratamiento es una de las principales variables en la cual los servicios de salud pueden intervenir para aumentar la efectividad de la medicación. El objetivo del estudio es evaluar la relación coste-efectividad de la adhesión inicial a la terapia ARV e identificar factores que influencien los costes y la evolución global del paciente.

Métodos: Estudio prospectivo con individuos infectados por el VIH reclutados, después de la primera prescripción de ARV, en dos servicios públicos de referencia para el tratamiento del VIH/Sida en Brasil. La no-adhesión fue definida como la utilización $<95 \%$ de la cantidad prescrita durante los 3 días anteriores a la primera visita de seguimiento. El ratio coste-efectividad se ha calculado para el grupo adherente y no-adherente. Se han empleado modelos de regresión lineal múltiple y logística binaria en el análisis univariante y multivariante.

Resultados: Un 79,7\% de los 197 participantes fue adherente al tratamiento ARV y el ratio coste-efectividad obtenido para este grupo (2.667,66 euros) fue menor que el observado entre los no adherentes. El análisis multivariante $(\mathrm{p}<0,05)$ mostró que la adhesión a la terapia ARV y el uso de inhibidores de la proteasa en el tratamiento estaban asociados con mayor coste directo medio de la atención. La evolución global favorable estuvo asociada con estar asintomático, tener linfocitos TCD4+>200 cels/mm3 y ser adherente al tratamiento ARV.

Correspondencia:

Francisco de Assis Acurcio

Rua Vinicius de Moraes, 83 Apto 1002

CEP:30.380-520 - Belo Horizonte

Minas Gerais. Brasil.

Correo eletrónico: acurcio@medicina.ufmg.br

Subvención: PN-DST/AIDS /Brasil/UNESCO/914 BRA

3014/521-02

\section{ABSTRACT \\ Cost-Effectiveness of Initial Adherence to Antiretroviral Therapy among HIV Infected Patients in Belo Horizonte, Brazil}

Background: In Brazil, there is free antiretroviral (ARV) distribution to patients with HIV disease by the National Health System. Adherence to the treatment is one of the main factors that health services can manage to increase the effectiveness of the ARV medication. The aim of this study is to evaluate the cost-effectiveness of initial adherence to the ARV therapy and to identify factors that influence patient's costs and global evolution.

Methods: Prospective study of HIV infected patients receiving their first ARV prescription in two public referral centers to HIV/Aids, Brazil. The non-adherence was defined as the intake of $<95 \%$ of the prescribed doses for 3 days prior to the first follow-up visit. A cost-effectiveness ratio was calculated for either groups, the adherent and the non-adherent. Univariate and multivariate analysis were performed using multiple linear and binary logistic regression models.

Results: $79.7 \%$ of the 197 participants was adherent to the ARV treatment and the ratio cost-effectiveness obtained for this group $(2,667.67$ euros) was smaller than the ratio observed among the non-adherent group. Multivariate analysis $(\mathrm{p}<0.05)$ showed that adherence to ARV therapy and use of protease inhibitors in the treatment were associated with higher direct cost of care. The favorable global evolution was associated with being asymptomatic, TCD4+ linphocyte count $>200$ cels $/ \mathrm{mm} 3$ and be adherent to the ARV treatment.

Conclusions: The observed results supported the ARV therapy as a cost-effective intervention. However, low adherence increases 
Conclusiones: Los resultados observados sostienen la terapia ARV como una intervención coste-efectiva. Sin embargo, la baja adhesión aumenta los riesgos de fallo terapéutico y de progresión de la enfermedad, que resultan en un impacto negativo sobre la relación coste-efectividad de la terapia ARV.

Palabras clave: VIH. Sida. Evaluación económica. Coste-efectividad. Terapia antirretroviral. Brasil the risk of therapeutic failure and illness progression that results in a negative impact on the cost-efectiveness of the ARV therapy.

Key words: HIV. Aids. Economic evaluation. Cost-effectiveness. Antiretroviral therapy. Brazil.

\section{INTRODUCCIÓN}

Aproximadamente entre 35,9 y 44,3 millones de personas eran portadores del virus de la inmunodeficiencia humana (VIH) en $2004^{1}$. La epidemia de VIH/Sida continúa siendo uno de los principales problemas de salud pública, a pesar de los avances en el conocimiento de esta enfermedad observados en los últimos años. En los países de ingresos bajos o medianos vive un 90\% de la población mundial infectada, no obstante, únicamente alrededor de un $10 \%$ tiene acceso a los medicamentos desarrollados por la industria farmacéutica ${ }^{1}$. El control la propagación del VIH/Sida se considera como la principal prioridad y la más costeefectiva entre un conjunto de propuestas para enfrentarse a los diez mayores desafíos globales contemporáneos ${ }^{2}$.

En Brasil, una de las principales estrategias para combatir la epidemia es la distribución de los medicamentos antirretrovirales (ARV) a los personas portadoras del VIH que necesiten tratamiento. En el país existen aproximadamente 600.000 personas infectadas y cerca de 160.000 ya hacen uso de ARV. Los gastos nacionales en ARV han crecido progresivamente, representando US $\$ 258,7$ millones en $2004^{3}$. Varios indicadores muestran el efecto positivo de esa política de acceso universal. Entre 1996 y 2002, tuvo lugar una reducción de la mortalidad, una disminución de las estancias hospitalarias y una reducción de la incidencia de infecciones oportunistas ${ }^{4}$.

Los resultados alcanzados en diversos estudios del tipo coste-efectividad de la terapéutica antirretroviral de gran actividad
(TARGA), tanto en los EUA como en Europa, indican que estos tratamientos ofrecen unos buenos resultados en comparación con los recursos invertidos ${ }^{5}$.

Sin embargo, el uso irregular de la TARGA puede propiciar el desarrollo de virus VIH resistentes, haciendo que una estricta adhesión al régimen prescrito sea indispensable para la efectividad del tratamiento ${ }^{6,7}$. Una baja adhesión puede conllevar futuras limitaciones terapéuticas para el paciente y una amenaza para la salud pública ${ }^{8-10}$. La noadhesión es la causa más común del fallo del tratamiento y es la principal variable en la cual los servicios de salud pueden intervenir directamente para aumentar la efectividad de la medicación. Se ha demostrado que intervenciones clínicas para incrementar la adhesión a la TARGA son coste-efectivas ${ }^{11}$. Entre los factores que pueden contribuir a la no adhesión se encuentran el aumento de la complejidad del tratamiento, la comprensión insuficiente del uso de los medicamentos, así como la falta de información sobre los riesgos de la no adhesión ${ }^{12,13}$. El coste del tratamiento ARV es demasiado alto y la no adhesión al mismo tiene gran impacto sanitario, lo cual puede contribuir a comprometer la rentabilidad social de unos recursos escasos que son dirigidos al combate de la epidemia.

En Brasil la epidemia de VIH/Sida es uno de los problemas de salud más importantes, con 362.364 casos de sida registrados hasta junio de $2004^{14}$. En este contexto, es fundamental la realización de estudios que analicen la evolución de los costes, la eficiencia y la efectividad de las nuevas formas de tratamiento. Los resultados obtenidos por estas evaluaciones pueden contribuir a una mejor 
distribución de recursos y a un mayor nivel de bienestar de las poblaciones infectadas por el VIH.

Los objetivos de este estudio son los siguientes: 1 . comparar en grupos de pacientes (adherentes y no adherentes en el inicio del tratamiento antirretroviral) la utilización de servicios, su evolución de la enfermedad y los costes directos de la atención a la salud; 2. evaluar la relación coste-efectividad de la adhesión inicial a la TARGA; y 3. determinar la influencia que sobre los costes y sobre la efectividad generan determinados factores relativos a las características sociodemográficas, clínicas y asistenciales de los participantes.

\section{SUJETOS Y MÉTODOS}

Población del estudio: Esta investigación forma parte del Proyecto ATAR ${ }^{15}$, un estudio prospectivo para verificar la adhesión a la TARGA en individuos infectados por VIH, en Belo Horizonte, Brasil. La población objeto del estudio está constituida por individuos adscritos a dos servicios públicos de salud de referencia para el VIH/Sida (CTR/DIP Orestes Diniz y Hospital Eduardo de Menezes). Ambos centros están autorizados para realizar la primera prescripción de antirretrovirales. Los participantes fueron entrevistados después de la primera entrega de los medicamentos y en visitas posteriores. El reclutamiento tuvo lugar desde mayo de 2001 hasta mayo de 2002. El período máximo de seguimiento fue establecido en doce meses después de la admisión. Los criterios de elegibilidad adoptados por el Proyecto incluyeron los individuos con infección por el VIH confirmada, con edad superior a 18 años, hasta entonces sin ningún tipo de tratamiento, que cumplían con los criterios del Ministerio de Salud brasileño para iniciar la TARGA, que recibieron ARV en uno de los servicios de referencia y que consintieron en participar en el estudio. Además de los criterios de inclusión ya mencionados, para el análisis coste-efectividad se han añadido los siguientes: individuos que retornaron para la primera visita de seguimiento después de la admisión, que tenían información sobre su evolución inmunológica (linfocitos TCD4+ en la primera y en la última consulta médica) y que recogieron ARV de forma regular en el dispensario durante un periodo mínimo de 9 meses. El perfil del estudio (figura 1), indica que del total de pacientes atendidos en el Proyecto ATAR ( $\mathrm{n}=503)$, un $39,2 \%$ no fueron incluidos en este estudio porque o no cumplían los criterios de elegibilidad, o se negaron a participar en el estudio, o se trataba de mujeres embarazadas o sólo respondieron a la entrevista inicial. Entra las 306 personas elegibles (un 60,8\%), es decir, aquéllos que retornaron por lo menos una vez a recoger medicación, 197 cumplían con los criterios de selección para el análisis coste-efectividad.

Los participantes en el estudio fueron divididos en dos grupos de acuerdo con la adhesión al tratamiento ARV. La adhesión inicial al tratamiento ARV fue definida como la utilización de un mínimo del 95\% de la cantidad prescrita por el médico, según auto-declaración, durante los tres días anteriores a la primera visita de seguimiento después de la admisión.

Recogida de datos: El proyecto fue aprobado por el Comité de Ética en Investigación de la Universidad Federal de Minas Gerais. La recogida de los datos fue realizada mediante instrumentos previamente probados y después de la capacitación de los investigadores. Se recogieron datos sobre variables socio-demográficas, variables relacionadas con el tratamiento ARV y con la utilización de servicios (consultas, derivaciones a especialista, hospitalización), variables clínicas (diagnósticos registrados en prontuario) y pruebas de laboratorio (linfocitos T-CD4+ y otras).

Medida de efectividad y costes: Se definió la efectividad como la probabilidad de 
Figura 1

Perfil del estudio

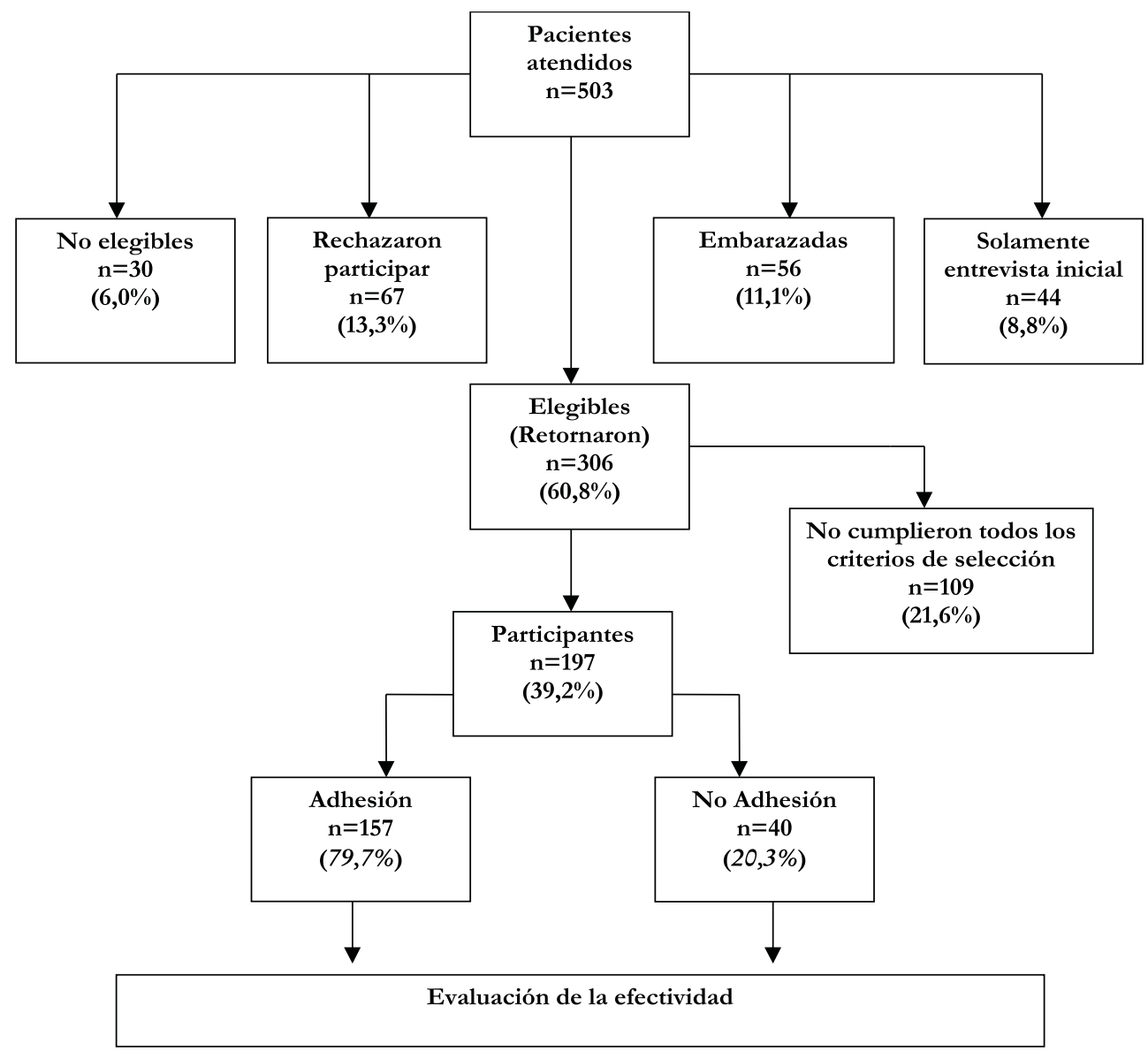

De los 503 pacientes atendidos, un 30,4\% $(n=153)$ fueron no elegibles, rechazaron participar o se trataba de mujeres embarazadas, mientras que un $8,8 \%$ solamente comparecieron a la entrevista inicial. Los 306 pacientes restantes eran candidatos potenciales para el análisis coste-efectividad, pero $109(21,6 \%)$ no cumplían tampoco con todos los criterios de inclusión. Así, fueron incluidos en el estudio 197 pacientes (adherentes: 157 y no adherentes: 40).

tener una respuesta satisfactoria al tratamiento, entendida como la ocurrencia simultánea de 3 condiciones durante el periodo de seguimiento: a. Evolución inmunológica favorable, considerándose las siguientes categorías del número de linfocitos TCD4+: 1) $\left.>500 \mathrm{cels} / \mathrm{mm}^{3}, 2\right)$ entre 200-500 cels $\left./ \mathrm{mm}^{3}, 3\right)<200$ cels $/ \mathrm{mm}^{3}$. Los valores del examen más próximo al inicio del tratamiento fueron comparados con los del último examen registrado en el periodo de seguimiento. La permanencia en las categorías 1 y 2, o la migración a una categoría cuantitativamente superior fueron consideradas como evolución inmunológica favorable. No se evaluó la carga viral porque no fue realizada por la mayoría de los participantes; b. Ausencia de hospitalización; c. Ausencia de nuevo diagnóstico de evidencia de inmunodeficiencia grave (Sida). 
El análisis de costes adoptó la perspectiva del financiador público y se limitó al análisis de los costes directos de la atención a la salud. Los costes de las actividades protocolizadas para la atención al portador de VIH y registradas en la historia clínica individual, en el periodo de 9 meses después de la primera entrega de ARV, se calcularon de la forma siguiente: a. Coste del régimen ARV prescrito según cada dispensación, calculado a partir de los precios unitarios de adquisición de los medicamentos por el Ministerio de Salud brasileño; b. Costes unitarios de procedimientos calculados a partir de la tabla de precios aplicada por el Sistema Único de Salud; c. Costes hospitalarios calculados a partir del coste medio de internamiento por diagnóstico específico, según el Sistema de Información Hospitalaria del Sistema Único de Salud.

El coste total de los ARV fue calculado como la suma de los costes de cada dispensación realizada para cada paciente. Para las demás categorías de utilización de servicios el coste total fue calculado como el producto del número de procedimientos o internamientos por el coste unitario de cada uno de estos servicios. Los costes fueron obtenidos a partir de los precios públicos aplicados el año 2002. Los valores están expresados en Reais ( $\mathrm{R} \$)$, unidad monetaria brasileña ( $\mathrm{R} \$$ $1,00=0,35 €)$.

Análisis estadístico: Se realizó un análisis de las características de los individuos elegibles para el estudio, comparándose participantes y no participantes. Los grupos de pacientes adherentes y no adherentes al inicio del tratamiento fueron comparados con relación a la utilización de servicios, evolución de la enfermedad y costes de la atención sanitaria. Los resultados fueron expresados como media \pm desviación estándar o porcentajes en las categorías correspondientes. Para comparaciones entre grupos fueron utilizados testes del Chi-cuadrado, T de Student o Mann-Whitney. En el análisis estadístico se adoptó un nivel de significación del 5\%.
Para evaluar la relación coste-efectividad de la adhesión a la terapia ARV, los indicadores se calcularon tanto para el grupo adherente como para el no adherente. La ratio de coste-efectividad media para cada grupo, comparada con la ausencia de terapia, se calculó dividiendo los costes directos relacionados entre el número de pacientes con respuesta satisfactoria al tratamiento en el periodo de estudio.

Para determinar la influencia que sobre los costes y sobre la efectividad generan determinados factores, se han empleado las siguientes variables explicativas: edad, sexo, raza, estado civil, escolaridad, renta individual y familiar, seguro privado de salud, clasificación clínica inicial, nivel inicial de linfocitos TCD4+, adhesión al tratamiento ARV y los inhibidores de la proteasa en el tratamiento ARV inicial. El análisis utilizado para estimar la relación de una o más variables explicativas con los costes directos de la atención ha sido la regresión lineal múltiple. Se ha diseñado un modelo máximo con todas las variables y se ha realizado la supresión secuencial de acuerdo con la importancia estadística. Se han mantenido en el modelo final aquellas variables con $\mathrm{p}<0,05$. Asimismo, se ha estimado un modelo de regresión logística binaria para identificar los factores que influenciaron la evolución global (efectividad) del paciente. En el análisis univariante, el riesgo relativo con intervalo de confianza del $95 \%$ fue utilizado para estimar la importancia de la asociación entre evolución global y cada una de las variables explicativas. El efecto independiente de las variables seleccionadas sobre la evolución global fue evaluado utilizando el mismo método en el análisis multivariante. Las variables asociadas estadísticamente con la evolución global en el análisis univariante $(\mathrm{p} \leq 0,20)$ fueron incluidas en el modelo multivariante.

Para el tratamiento estadístico de los datos se han utilizado el software SPSS $® 11.5 \mathrm{y}$ EPIINFO® 3.3. 


\section{RESULTADOS}

Análisis descriptivo: En la admisión al estudio, los 197 participantes y 109 no participantes presentaron características sociodemográficas, clínicas y asistenciales muy semejantes (tabla 1). Sólo se observaron diferencias estadísticamente significativas con relación a la edad (los no participantes eran más jóvenes) y con la ocurrencia de hospitalización (mayor proporción entre no participantes).

Los participantes en el estudio tenían una

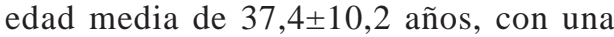
mayor proporción de hombres (un 62,9\%), no blancos (un 75,3\%) y solteros (un $47,7 \%)$. Los niveles de escolaridad y de renta eran $7,4 \pm 3,4$ años y $\mathrm{R} \$ 373,79 \pm 545,65$, respectivamente y sólo un 59,2\% declararon tener renta individual. El 25,4\% disponía de seguro privado de salud. Las características clínicas indican que un $60,4 \%$ ya presenta- ban un número de linfocitos TCD4+ $\leq$ 200 cels $/ \mathrm{mm}^{3}$ cuando iniciaron el tratamiento. Cerca de un $30,0 \%$ de ellos ya poseía al menos un diagnóstico de enfermedades relacionadas con el sida. Durante el periodo de seguimiento, el número medio de visitas médicas fue de $6,9 \pm 4,0$ entre todos los participantes y un $10,7 \%$ de ellos fue hospitalizado. La ocurrencia de no adhesión fue relatada por 40 participantes (un 20,3\%) en la primera entrevista después de la admisión.

Resultados clínicos: El período de seguimiento de los resultados clínicos fue de 1 año. Durante este periodo se observó la ocurrencia de nuevos diagnósticos relacionados con el Sida en una misma proporción (un $27 \%$ ) para ambos grupos de pacientes adherentes y no adherentes (tabla 2). Aproximadamente un tercio de los pacientes en los dos grupos presentó una evolución inmunológica desfavorable. De los pacientes no adhe-

Tabla 1

Características de los individuos elegibles para el estudio, en la entrevista de entrada (baseline): Análisis de las pérdidas, Belo Horizonte (MG), 2001-2003

\begin{tabular}{|c|c|c|c|}
\hline Características & Participantes & No Participantes & Total \\
\hline \multicolumn{4}{|l|}{ Sócio-demográficas } \\
\hline Sexo (\% hombres) & $197(62,9)$ & $109(67,9)$ & $306(64,7)$ \\
\hline Edad, años ${ }^{\mathrm{a}}$ & $37,4 \pm 10,2$ & $34,8 \pm 8,9$ & $36,5 \pm 9,8$ \\
\hline Raza (\% afro-descendientes) & $190(75.3)$ & $104(73.1)$ & $294(74,5)$ \\
\hline Estado civil (\% soltero) & $197(47,7)$ & $109(45,0)$ & $306(46,7)$ \\
\hline Escolaridad, años & $7,4 \pm 3,4$ & $7,3 \pm 3,8$ & $7,3 \pm 3,5$ \\
\hline Renta individual el último mes (\% si) & $196(59,2)$ & $108(58,3)$ & $304(58,9)$ \\
\hline Renta individual media en Reais ${ }^{b}$ & $373,79 \pm 545,65$ & $393,45 \pm 706,84$ & $380,76 \pm 606,57$ \\
\hline Renta familiar el último mes (\% si) & $196(84,7)$ & $107(79,4)$ & $303(82,8)$ \\
\hline Seguro privado de salud (\% sí) & $197(25,4)$ & $109(21,1)$ & $306(23,9)$ \\
\hline \multicolumn{4}{|l|}{ Clínicas } \\
\hline Clasificación inicial (\% sida) & $197(29,9)$ & $98(33,7)$ & $295(31,2)$ \\
\hline Numero de linfocitos TCD4+ $\left(\%<200 \mathrm{cels} / \mathrm{mm}^{3}\right)$ & $197(60,4)$ & $75(49,3)$ & $272(57,4)$ \\
\hline \multicolumn{4}{|l|}{ Asistenciales } \\
\hline Visitas médicas & $6,9 \pm 4,0$ & $7,8 \pm 5,5$ & $7,2 \pm 4,6$ \\
\hline Adhesión al tratamiento ARV (\% no) & $197(20,3)$ & $109(23,9)$ & $306(21,6)$ \\
\hline Hospitalización (\% sí) & $196(10,7)$ & $103(28,2)$ & $299(16,7)$ \\
\hline
\end{tabular}

Los resultados se presentan como media desviación estándar o como porcentaje de individuos en la categoría correspondiente. Las cifras totales varían debido a datos no disponibles.

a $\mathrm{P}$-value $<0,05$

b $1 \mathrm{R} \$=0,35 €$ 
Tabla 2

Resultados clínicos, efectividad y utilización de servicios entre los participantes, Belo Horizonte (MG), 2001-2003

\begin{tabular}{|lccc|}
\hline & $\begin{array}{c}\text { Adherentes } \\
(\mathbf{n}=\mathbf{1 5 7})\end{array}$ & $\begin{array}{c}\text { No Adherentes } \\
(\mathbf{n}=\mathbf{4 0})\end{array}$ & $\mathbf{p}$ \\
\hline Resultados clínicos (1 año de seguimiento) & & & \\
$\begin{array}{l}\text { Diagnósticos nuevos relacionados a la Sida: } \\
\text { Pacientes, n (\%) }\end{array}$ & $43(27,4)$ & $11(27.5)$ & $0,99^{\mathrm{a}}$ \\
Numero de episódios & $0,48 \pm 0,95$ & $0,53 \pm 1,01$ & $0,78^{\mathrm{b}}$ \\
Evolución inmunológica desfavorable, n (\%) & $57(36,3)$ & $13(32,5)$ & $0,65^{\mathrm{a}}$ \\
& $13(8,3)$ & $8(20,0)$ & $0,03^{\mathrm{a}}$ \\
Ocurrencia de hospitalización, n(\%) & & & \\
Efectividad (1 año de seguimiento) & $76(48,4)$ & $14(35,0)$ & $0,13^{\mathrm{a}}$ \\
Evolución global favorable, n(\%) & & & \\
Utilización de servicios (9 meses de seguimiento) & $5,75 \pm 3,25$ & $7,95 \pm 5,61$ & $<0,01^{\mathrm{c}}$ \\
Número total de visitas médicas & & $5,70 \pm 2,75$ & $0,03^{\mathrm{b}}$ \\
Número de visitas al clínico / especialista $\quad$ en enfermedades infecciosas & $4,70 \pm 2,55$ & $2,25 \pm 3,36$ & $0,01^{\mathrm{c}}$ \\
Número de visitas a otros especialistas & $1,05 \pm 1,62$ & $15,00 \pm 11,39$ & $0,40^{\mathrm{c}}$ \\
Número total de pruebas de laboratorio & $15,06 \pm 8,22$ & $8,52 \pm 1,48$ & $0,02^{\mathrm{b}}$ \\
Número de entregas de antiretrovirales & $9,17 \pm 1,56$ & $0,25 \pm 0,54$ & $0,03^{\mathrm{c}}$ \\
\hline Número de hospitalizaciones & $0,08 \pm 0,27$ & & \\
\hline
\end{tabular}

Resultados son presentados como media \pm desviación estándar o como porcentaje de individuos en la categoría correspondiente. ${ }^{\mathrm{a}}$ test del Chi-cuadrado; ${ }^{\mathrm{b}}$ test t de Student; ${ }^{\mathrm{c}}$ test de Mann-Whitney.

rentes fueron hospitalizados el 20,0\%, y de los adherentes el 8,3\% ( $\mathrm{p}=0,03)$.

Utilización de servicios: En los 9 meses de seguimiento para la utilización de servicios, se verificó (tabla 2) una mayor frecuencia de visitas médicas entre los pacientes no adherentes que entre los adherentes $(\mathrm{p}<0,05)$. El número medio de hospitalizaciones también fue significativamente mayor entre los no adherentes $(0,25 \pm 0,54)$ que entre los adherentes $(0,08 \pm 0,27)$ $(\mathrm{p}=0,03)$. Los dos grupos presentaron un número medio de 15 pruebas de laboratorio. En este periodo, el número medio de entre-

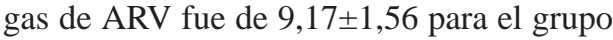

adherente y de $8,52 \pm 1,48$ para el grupo no adherente, siendo esta diferencia significativa del punto de vista estadístico $(\mathrm{p}=0,02)$.

\section{Costes directos de la atención sanitaria:}

El grupo no adherente mostró costes medios más elevados en lo que se refiere a las visitas médicas, derivaciones a los especialistas y estancias hospitalarias (tabla 3). No se encontraron diferencias significativas con relación a los costes de pruebas de laboratorio. El valor medio de la combinación de ARV en cada entrega fue de $R \$ 375,92 \pm 180,49$. El coste de ARV fue mayor en el grupo adherente, pero la diferencia no fue significativa $(\mathrm{p}=0,16)$. En su conjunto, el coste directo medio por 
Costes directos medios por paciente y ratio coste-efectividad, según adhesión, Belo Horizonte (MG), 2001-2003

\begin{tabular}{|c|c|c|c|c|c|c|}
\hline \multirow{2}{*}{ Categorías } & \multicolumn{2}{|c|}{ Adherentes } & \multicolumn{3}{|c|}{ No Adherentes } & \multirow{2}{*}{ p-valor } \\
\hline & $\begin{array}{l}\text { Coste por } \\
\text { categoria }\end{array}$ & $\begin{array}{l}N^{\circ} \text { de Eventos / } \\
\text { Pacientes }\end{array}$ & $\begin{array}{c}\text { Coste por paciente, euros } \\
\text { (reais) }\end{array}$ & $\begin{array}{c}\mathbf{N}^{0} \text { de Eventos } \\
\text { / Pacientes }\end{array}$ & $\begin{array}{c}\text { Coste por paciente, euros } \\
\text { (reais) }\end{array}$ & \\
\hline $\begin{array}{l}\text { Visitas clinico / especialista en } \\
\text { enfermedades infecciosas }\end{array}$ & $0,92(2,62)$ & $739 / 156$ & $4,32(12,33)$ & $228 / 40$ & $5,23(14,93)$ & $0,03^{\mathrm{a}}$ \\
\hline Visitas otros especialistas & $1,14(3,25)$ & $165 / 67$ & $1,20(3,42)$ & $90 / 25$ & $2,56(7,31)$ & $0,01^{\mathrm{b}}$ \\
\hline Linfocitos CD4/CD8 & $5,25(15,00)$ & $390 / 157$ & $13,04(37,26)$ & $86 / 40$ & $11,29(32,25)$ & $0,06^{\mathrm{a}}$ \\
\hline Carga viral & $6,30(18,00)$ & $270 / 141$ & $10,84(30,96)$ & $56 / 33$ & $8,82(25,20)$ & $0,07^{\mathrm{a}}$ \\
\hline Otras pruebas laboratorio & - & $1705 / 146$ & $16,04(45,82)$ & $458 / 35$ & $15,43(44,09)$ & $0,80^{\mathrm{a}}$ \\
\hline Estancias hospitalarias & - & $13 / 13$ & $24,83(70,95)$ & $10 / 8$ & $76,11(217,45)$ & $0,02^{\mathbf{b}}$ \\
\hline Antiretrovirales & - & $-\ldots$ & $1221,10(3488,85)$ & -- & $1070,77(3059,34)$ & $0,16^{\mathrm{a}}$ \\
\hline Coste directo medio por paciente & & & $\mathbf{1 2 9 1 , 3 5}(3689,58)$ & & $1190,20(3400,58)$ & $\mathbf{0 , 3 0}{ }^{\mathrm{a}}$ \\
\hline$[\mathrm{IC} 95 \%]^{\mathrm{c}}$ & & & {$[1191,56-1391,15]$} & & {$[1029,01-1351,39]$} & \\
\hline Coste total & & & $202742,68(579264,80)$ & & $47608,14(136023,25)$ & \\
\hline Evolución global favorable & & & 76 & & 14 & \\
\hline
\end{tabular}

Costes son expresados en Euros (y en Reais) para el año de 2002. Coste por categoría indica el coste unitario medio estimado (eg coste de una visita médica). No. de eventos/pacientes, número de unidades de la categoría correspondiente y número de pacientes activos en aquella categoría, respectivamente. El coste medio por paciente para una dada categoría fue calculado como el producto del coste unitario por categoría veces el número de eventos dividido por el número total de pacientes en el grupo (Adherentes=157 o No Adherentes=40). La ratio coste-efectividad en cada grupo fue calculada dividiéndose el coste directo total por el número de pacientes con evolución global favorable en el período de acompañamiento.a Test t; b Test Mann-Whitney; c [IC 95\% CI], Intervalo de confianza 95\%. 
paciente fue un poco más elevado (un $8,5 \%$ ) entre los adherentes, pero esa diferencia tampoco fue estadísticamente significativa $(\mathrm{p}=0,30)$. Los ARV representaron un 94,6\% del coste medio entre los adherentes y un $90,0 \%$ de este coste entre los no adherentes.

En el modelo de regresión lineal múltiple final obtenido para explicar el coste directo (tabla 4) resulta significativa la contribución de las variables: adhesión al tratamiento ARV y la utilización de inhibidores de la proteasa en el tratamiento ARV inicial $\left(\mathrm{F}=116,457 ; \mathrm{p}=0,000 ; \mathrm{R}=0,746 ; \mathrm{R}^{2}=0,556\right.$; $\left.\beta=1950,70 ; \beta_{1}=496,43 ; \beta_{2}=2606,01\right)$. Se ha llevado a cabo una validación del modelo estimado y se ha verificado que no existe autocorrelación entre los residuos, éstos presentan una distribución normal y no existen problemas de multicolinealidad entre las variables explicativas. Según este modelo, los pacientes adherentes a los ARV suponen un coste directo medio de $\mathrm{R} \$$ 496,43 más que los pacientes no adherentes, una vez controlado el efecto de las variables explicativas seleccionadas. Además, los pacientes con inhibidores de la proteasa en el tratamiento con ARV suponen un coste directo medio de $\mathrm{R} \$ 2606,01$ más que los que no tienen este tratamiento.

Efectividad: Los indicadores de efectividad se han calculado a partir de los resulta- dos clínicos. De los 197 participantes, 90 (un $45,7 \%$ ) presentaron evolución global favorable durante el seguimiento (Tabla 3). La respuesta satisfactoria fue más frecuente entre los adherentes.

La tabla 5 muestra los resultados de la estimación de un modelo de regresión logística en el que la evolución favorable es la variable dependiente. La evaluación del ajuste del modelo final mostró adecuación (Test de Hosmer y Lemeshow (8g.1.) = $13,719 ; \mathrm{p}=0,089)$. Los individuos asintomáticos al inicio del seguimiento y adherentes al tratamiento ARV tuvieron una probabilidad 3 veces mayor de presentar una evolución favorable en el período observado, comparado con sus grupos de referencia $(\mathrm{p}<$ $0,01)$. Además, los individuos con niveles de linfocitos TCD4+ superiores a 200 cels/ $\mathrm{mm}^{3}$ al inicio del tratamiento, tuvieron una probabilidad 4,3 veces mayor de evolucionar favorablemente que aquéllos con bajos niveles de estas células.

Ratio coste-efectividad: El coste total de la atención a la salud para el grupo adherente fue de $\mathrm{R} \$ 579.264,80$. De los 157 individuos pertenecientes a este grupo, 76 (un $48,4 \%$ ) presentaron una evolución global favorable. Para los 40 individuos del grupo no adherente se registró un coste de la atención de $\mathrm{R} \$ 136.023,25$ y 14 (un $35,0 \%$ )

Tabla 4

Modelo final de la regresión lineal múltiple. Variable dependiente: coste directo total por paciente. Belo Horizonte (MG), 2001-2003

\begin{tabular}{|lccccc|}
\hline & & F & Significación $\mathbf{~}$ & \\
\hline $\mathrm{R}$ múltiple & 0,746 & & & & \\
$\mathrm{R}^{2}$ & 0,556 & & 116,457 & 0,000 & \\
$\mathrm{R}^{2}$ ajustada & 0,551 & & & & \\
Variables & $\mathbf{B}$ & $\mathbf{S E ~} \boldsymbol{B}$ & $\mathbf{T}$ & Significación t & IC 95\% \\
Constante & 1950,70 & 211,48 & 9,224 & 0,000 & $1533,48-2367,91$ \\
ADHESIÓN & 496,43 & 214,11 & 2,319 & 0,022 & $74,03-918,83$ \\
IP & 2606,01 & 171,75 & 15,173 & 0,000 & $2267,18-2944,83$ \\
\hline
\end{tabular}

IP: Inhibidor de protease en el esquema ARV inicial; ADHESIÓN: adhesión al tratamiento ARV 
Modelo final $^{1}$ de la regresión logística binaria. Variable dependiente: evolución global del paciente. Belo Horizonte (MG), 2001-2003

\begin{tabular}{|lcc|}
\hline \multicolumn{1}{|c|}{ Características } & $\begin{array}{c}\text { Riesgo Relativo } \\
\text { (IC 95\%) }\end{array}$ & p \\
\hline Clasificación clínica (asintomático) & $3,08(1,63-5,83)$ & 0,001 \\
Linfocitos TCD4+ $\left(>200\right.$ cels $\left./ \mathrm{mm}^{3}\right)$ & $4,29(2,17-8,49)$ & 0,000 \\
Adhesión al tratamiento (sí) & $3,15(1,34-7,42)$ & 0,009 \\
Constante & 0,107 & 0,000 \\
\hline
\end{tabular}

1 Test de Hosmer y Lemeshow (8g.1.): 13,719; $\mathrm{p}=0,089$

pacientes con respuesta satisfactoria. El ratio coste-efectividad obtenido para el grupo adherente al tratamiento ARV (R \$ 7.621,91) comparado con la no terapia fue menor que el observado entre los no adherentes ( $\mathrm{R} \$$ 9.715,95) (tabla 3).

\section{DISCUSIÓN}

El perfil sociodemográfico de los individuos estudiados permite sospechar que habría serios problemas de accesibilidad económica a los ARV si estos medicamentos no fueran distribuidos de forma gratuita por el Sistema Único de Salud. Los niveles de renta eran bajos y se pudo constatar que el valor medio de cada dispensación de ARV correspondía casi al mismo valor de la renta media individual. Esta situación pone de manifiesto la necesidad de realizar intervenciones coste-efectivas en la organización de la atención al individuo infectado por el VIH como forma de garantizar la continuidad de esta atención de manera universal, como viene siendo practicado en Brasil.

Al inicio del tratamiento ARV, la mayoría de los participantes ya presentaban un bajo número de linfocitos TCD4+ y un tercio de ellos ya poseía al menos un diagnóstico de Sida. Estas elevadas proporciones son preocupantes pues indican un inicio tardío de la terapia ARV que, por su parte, puede estar relacionado con la dificultad de acceso a los servicios de salud y/o con la desinformación sobre la importancia del tratamiento precoz. El Ministerio de Salud brasileño recomienda una mayor frecuencia en la evaluación de la necesidad de inicio de la terapia de pacientes asintomáticos con recuento de linfocitos TCD4+ entre 200 y 350 cels $/ \mathrm{mm}^{3}$. Niveles de linfocitos TCD4+ $<200$ cels $/ \mathrm{mm}^{3}$ están asociados a un aumento acentuado de la incidencia de infecciones oportunistas y también a una respuesta terapéutica menos duradera $^{13}$. Por eso, el recuento inicial de linfocitos TCD4+ ha sido considerado uno de los más importantes determinantes de los costes, beneficios clínicos y coste-efectividad entre infectados por el $\mathrm{VIH}^{17}$. Se han observado costes de tratamiento más elevados en pacientes que se presentaron tardíamente en los servicios de salud ${ }^{16}$. El presente estudio no encontró asociación significativa del nivel de linfocitos TCD4+ con los costes directos de la atención, sin embargo, los pacientes que iniciaron el tratamiento con niveles más elevados de linfocitos, y también aquellos asintomáticos, presentaron claramente una mayor probabilidad de evolución global favorable. A pesar de todo, debido a la incertidumbre sobre los efectos adversos a largo plazo, existe controversia en cuanto al momento apropiado de iniciar la TARGA en pacientes con células TCD4+ entre 200 cels $/ \mathrm{mm}^{3}$ y 350 cels $/ \mathrm{mm}^{3}$. La tera- 
pia precoz ha sido respaldada por estudios recientes como una alternativa adecuada y coste-efectiva $^{18,19,20}$. Adicionalmente, cabe resaltar que la decisión de inicio de la TARGA en estos pacientes debe tener en cuenta ciertas características individuales tales como la motivación para el tratamiento, la capacidad de adhesión y la existencia de comorbilidades $^{13}$.

Uno de cada cinco participantes fue no adherente al inicio del tratamiento, y la no adhesión estuvo asociada con una mayor utilización de servicios ambulatorios y hospitalarios. Una mayor utilización de servicios ha sido relacionada con peores resultados en la salud de individuos infectados por el $\mathrm{VIH}^{21,22}$. Después de la introducción de la TARGA, en 1996, se observó un declive de la incidencia de infecciones oportunistas y la correspondiente reducción en el uso y en los costes de cuidados hospitalarios $3,5,23,24$. En el presente estudio, aunque los costes directos de la utilización de servicios sean menos elevados entre los individuos adherentes, se verifica que el coste directo medio por paciente no presentó diferencia significativa entre los grupos. Esto se explica principalmente por el elevado coste relativo de los ARV. Otro estudio también identificó los medicamentos como principales responsables de los costes directos de atención (un 82,2\%) para pacientes portadores del VIH en España, en $2003^{25}$. En nuestro estudio, las variables predictivas del coste directo total por paciente fueron: la adhesión al tratamiento y la presencia de inhibidores de la proteasa (IP) en el tratamiento ARV inicial. Un mayor número de entregas de ARV y su elevado coste relativo explica la influencia positiva de la adhesión al tratamiento sobre el coste total. Pero, es la presencia del IP en la terapia lo que supone el mayor incremento en el coste directo total por paciente. En esta situación, la inadecuada prescripción de los ARV puede haber inducido a una pérdida de eficiencia de los recursos disponibles. Un 47,7\% de los participantes recibieron IP ya en el tratamiento inicial. Sin embargo, los tratamientos con IP no deberían configurarse como tratamiento preferente ${ }^{13}$, pues los tratamientos con inhibidor de transcriptasa reversa no-análogo de nucleósido (ITRNN) son de posología más simple y tienden a presentar menos efectos adversos. Además, estudios con pacientes vírgenes de tratamiento presentan resultados equivalentes ${ }^{26,27,28}$ o más favorables con el uso de ITRNN $^{29}$. También en este estudio, iniciar la terapia ARV con IP no estuvo asociado a una mayor efectividad. Una importante heterogeneidad en la elección de tratamientos ARV, inconsistente con los protocolos terapéuticos, también fue observada en México resultando en un alto coste anual por pacien$\mathrm{te}^{30}$.

La adhesión a la TARGA ha sido asociada a resultados positivos en la salud de pacientes infectados por el VIH ${ }^{31,32}$. Resultados semejantes fueron encontrados en este estudio, donde individuos adherentes presentaron una probabilidad tres veces mayor de evolución favorable que los no adherentes. Así, aunque se observe un coste bastante fijo por paciente, la mayor efectividad derivada de la adhesión al tratamiento resultó en un menor ratio coste-efectividad entre los adherentes que entre no adherentes, comparando ambos grupos con la ausencia de terapia.

Estudios realizados en países desarrollados presentan resultados consistentes, con un ratio coste-efectividad estimado en cerca de U\$20.000/AVAC para la TARGA, comparado con la no terapia ${ }^{5}$. Otros estudios refuerzan también la consideración de la TARGA como una intervención coste-efectiva $^{33,34,35}$. Los regímenes terapéuticos ARV ofrecen una buena utilidad para los recursos utilizados en comparación con otras intervenciones. Aún con el alto coste de los ARV, la caída en la utilización hospitalaria y el incremento en la calidad de vida con la TARGA compensan el coste de los medicamentos ${ }^{32,36,37}$. Pero PHAIR et al. ${ }^{38}$ alertan que, con el transcurrir del tiempo, el éxito de la TARGA en prolongar la vida fue mayor 
que el éxito en reducir el coste y, por consecuencia, resulta un aumento del coste de la atención a lo largo de la vida del individuo, con aumentos no proporcionales en los gastos farmacéuticos. Sin embargo, siguen siendo necesarios análisis coste-efectividad más refinados sobre la TARGA. En el contexto de países de baja renta, será importante incluir los costes asociados con la utilización actual y las futuras consecuencias de estas intervenciones. Así, incluir las ganancias de productividad resultantes de la reducción de la morbididad y del incremento en la supervivencia de jóvenes puede hacer la TARGA más coste-efectiva en estos países. También la probabilidad de que la TARGA disminuya el riesgo de transmisión del VIH o la mayor motivación para realizar la prueba para el VIH a consecuencia de la disponibilidad de tratamiento efectivo son aspectos que deben ser considera$\operatorname{dos}^{5,39,40}$. Diversas intervenciones clínicas para mejorar la adhesión a la TARGA también aparecen como coste-efectivas. Un estudio evaluó la relación coste-efectividad de estas intervenciones en pacientes en diferentes fases de la enfermedad, eficacia de tratamiento y probabilidad de adhesión. Se encontró un ratio coste-efectividad inferior a US\$ 50.000/AVAC para intervenciones con coste mensual entre US\$ 100 y US\$ 500 , que conseguían aumentar la adhesión y reducir los fallos de supresión viral entre un $10 \%$ y un $50 \%{ }^{11}$.

Este estudio presenta algunas limitaciones que resulta conveniente reseñar. La principal de ellas es que aproximadamente un tercio de los individuos elegibles no pudieron ser incluidos en el análisis costeefectividad. El principal motivo de pérdida fue el no registro del nivel de linfocito TCD4+, principalmente al final del seguimiento, lo que imposibilitaba la medida de efectividad. La mayoría de las características eran semejantes entre participantes y no participantes, pero se observó mayor ocurrencia de hospitalización en este último grupo. Si se admite un sesgo de selección con menor participación de individuos más graves y si este grupo de individuos es, en su mayoría, no adherente, tal como ocurre con la población estudiada, entonces es posible que el ratio coste-efectividad para los no adherentes esté subestimado, hecho que no modifica las conclusiones generales del estudio. Otra limitación en la medida de la efectividad fue la constatación de que la mayoría de los pacientes tampoco tenía registro de carga viral en su historia clínica, en el período de seguimiento. Esa ausencia de registro puede significar dificultad de acceso a pruebas importantes para el seguimiento de la terapia ARV, como son el nivel de linfocito TCD4+ y la carga viral, o problemas relativos a la calidad del registro de la historia clínica.

Los resultados observados en este estudio están de acuerdo con los presentados en la literatura y que permiten identificar la TARGA como una intervención coste-efectiva que reduce la morbididad y la mortalidad relacionada al VIH. Sin embargo, para garantizar una respuesta satisfactoria al tratamiento son necesarios niveles elevados de adhesión. La baja adhesión aumenta los riesgos de fallo terapéutico y de progresión de la enfermedad, que resultan en un impacto negativo sobre el coste-efectividad de la TARGA. Así, la iniciativa del Sistema de Salud brasileño de distribuir los medicamentos ARV para el tratamiento de pacientes con VIH/Sida, que representa un gran esfuerzo social con costes crecientes, debe ser acompañada de mayor racionalidad en el uso de estos medicamentos además de seguimiento adecuado y soporte sistemático a los individuos para incrementar la adhesión y obtener una mayor efectividad del tratamiento. La adhesión satisfactoria a la TARGA debe ser considerada un proceso complejo y son necesarios más estudios para que comprendamos mejor las dificultades de cumplimiento del tratamiento, así como las consecuencias sanitarias y económicas que la no adhesión a la terapia ARV acarrea. 


\section{BIBLIOGRAFÍA}

1. Joint United Nations Programme on HIV/AIDS. AIDS epidemic update - December 2004 [capturado en 19/05/2005]. Se consigue en: http://www. unaids.org/ wad2004/report.html

2. News in brief. Fighting AIDS is best use of money, says cost-benefit analysis. Nature 2004; 429:592.

3. Brasil. Coordenação Nacional de DST e Aids. Resposta +: Atenção às pessoas que vivem com HIV e Aids. Brasília: Ministério da Saúde; 2005. [capturado en 09/08/2005]. Se consigue en: http://www. aids.gov.br/final/biblioteca/resposta/ resposta_2005.pdf.

4. Brasil. Coordenação Nacional de DST e Aids. Aids Números apontam estabilização de novos casos. Brasília: Ministério da Saúde; 2003. [capturado en 04/12/2003]. Se consigue en: http://www.aids. gov.br/imprensa/NoticiasImpressao.asp?NOTCod $=52007$

5. Yasdanpanah Y. Costes associated with combination antiretroviral therapy in HIV infected patients. J Antimicrob Chemother 2004; 53:558-61.

6. Mannheimer S, Friedland G, Matts J, Child C, Chesney M. The consistency of adherence to antiretroviral theraphy predicts biologic outcomes for human immunodeficiency virus-infected persons in clinical trials. Clin Infec Dis. 2002; 34:1115-21.

7. Metha S, Moore RD, Graham NMH. Potential factors affecting adherence with HIV therapy. AIDS 1997; 11:1665-70.

8. Poppa A, Davidson O, Deutsch J, Godfrey D, Fisher M, Head S et al. British HIV Association (BHIVA)/British Association for Sexual Health and HIV (BASHH) guidelines on provision of adherence support to individuals receiving antiretroviral therapy (2003). HIV Med. 2004; 5 Suppl 2:46-60.

9. Chesney MA. Factors affecting adherence to antiretroviral therapy. Clin Infect Dis 2000; 30 Suppl 2:171-6

10. Nemes MIB. Aderência ao tratamento por antiretrovirais em serviços públicos de saúde no Estado de São Paulo. Brasília: Ministério da Saúde, 2000. $172 \mathrm{p}$

11. Goldie SJ, Paltiel D, Weinstein MC, Losina E, Seage GR, Kimmel AD et al. Projecting the cost-effectiveness of aherence interventions in persons with human immunodeficiency virus infection. Am J Med 2003; 115:632-41.
12. Ceccato MGB, Acurcio FA, Bonolo PF, Rocha GM, Guimarães MD. Compreensão de informações relativas ao tratamento anti-retroviral entre indivíduos infectados pelo HIV. Cad Saúde Pública 2004; 20:1388-97.

13. Brasil. Coordenação Nacional de DST e Aids. Recomendações para a terapia anti-retroviral em adultos e adolescentes infectados pelo HIV-2004. Brasília: Ministério da Saúde; 2003. [capturado en 03/12/2003]. Disponible en: htpp://www. aids.gov.br/ final/biblioteca/adultos_2004/consenso.doc

14. Brasil. Coordenação Nacional de DST e Aids. Boletim Epidemiológico Aids e DST - Ano I n.01-Janeiro a Junho de 2004. Brasília: Ministério da Saúde; 2005. [capturado en 19/05/2005]. Disponible en: http://www.aids.gov.br/final/dados/ BOLETIM2.pdf

15. Bonolo PF, Cesar CC, Acurcio FA, Ceccato MGB, Pádua CAM, Guimarães MDC et al. Non-adherence among patients initiating antiretroviral therapy: a challenge for health professionals in Brazil. AIDS 2005; 19 (suppl 4): S 5-13.

16. Krentz HB, Auld MC, Gill MJ. The high cost of medical care for patients who present late $(\mathrm{CD} 4<200$ cels $/ \mu \mathrm{L})$ with HIV infection. HIV Med 2004; 5:93-8.

17. Freedberg KA, Losina E, Weinstein MC, Paltiel AD, Cohen CJ, Seage GR et al. The cost effectiveness of combination antiretroviral therapy for HIV disease. N Engl J Med 2001; 344:824-31.

18. Schackman BR, Freedberg KA, Weinstein MC, Sax PE, Losina E, Zhang H et al. Cost-effectiveness implications of the timing of antiretroviral therapy in HIV-infected adults. Arch Intern Med $2002 ; 162: 2478-86$

19. Mauskopf J, Kitahata M, Kauf T, Richter A, Tolson J. HIV antiretroviral treatment: early versus later. J Acquir Immune Defic Syndr 2005; 39:562-9.

20. Holmberg SD, Palella FJ, Lichtenstein KA, Havlir DV. The case for earlier treatment of HIV infection. Clin Infect Dis 2004; 39:1699-704.

21. Acurcio FA, Guimarães MDC. Uso de los servicios de salud y progresión al sida entre personas con infección por el VIH en Belo Horizonte (MG), Brasil. Rev Panam Salud Publica 1998; 4:331-40.

22. Acurcio FA, César CC, Guimarães MDC. Health care utilization and survival among patients with AIDS in Belo Horizonte (MG), Brasil. Reports in Public Health 1998; 14: 801-20. 
23. Chequer P, Cuchi P, Mazin R, Garcia Calleja JM. Access to antiretroviral treatment in Latin American countries and the Caribbean. AIDS 2002; 16 Suppl 3:S50-7.

24. Tramarin A, Campostrini S, Postma MJ, Calleri G, Tolley K, Parise N et al. A multicentre study of patient survival, disability, quality of life and cost of care: among patients with AIDS in northern Italy. Pharmaco Economics 2004; 22:43-53.

25. Lopez-Bastida J, Serrano-Aguilar P, Oliva J, Perestelo L. Direct and indirect costs in ambulatory patients living with HIV/Aids in Spain. Proceedings of the IHEA 5th World Congress; $2005 \mathrm{Jul}$ 10-13; Barcelona, Spain. Barcelona: UPF; 2005. p.327.

26. Bartlett JA, Johnson J, Herrera G et al. Initial therapy with abacavir + lamivudine $(\mathrm{ABC}+3 \mathrm{TC}) \mathrm{com}-$ bined with efavirenz (NNRTI), amprenavir/ritonavir (IP), or stavudine (NRTI): ESS40001 (CLASS). Proceedings of the XV International AIDS Conference; 2004 Jul 11-16; Bangkok, Thailand. Bangkok: 2004. Abstract TuPeB4544.

27. Van Leeuwen R, Katlama C, Murphy RL et al. Estudo atlantic: a randomized trial to study firstline combination therapy with or without a protease inhibitor in HIV-1-infected patients. AIDS 2003;17:987-99.

28. Podzamczer D, Ferrer E, Consiglio E et al. Estudo combine: a randomized clinical trial comparing nelfinavir or nevirapine associated to zidovudine/lamivudine in HIV-infected naïve patients (The combine study). Antiviral Ther 2002; 7:81-90.

29. Cooper D, Yeni P. Virological and immunological outcomes at 3 years following initiation of ART with regimens containing a NNRTI or PI or both: The INITIO trial. Proceedings of the 12th Conference on Retroviruses and Opportunistic Infections; 2005 Feb 22-25; Boston, USA. Boston; 2005. Abstract 165LB

30. Bautista S, Mane A, Bertozzi S. The economic impact of choice of antiretroviral therapy in the context of rapid scalling up access to treatment: Lessons from Mexico. Proceedings of the IHEA 5th World Congress; 2005 Jul 10-13; Barcelona, Spain. Barcelona: UPF; 2005. p.162.
31. Garcia de Olalla P, Knobel H, Carmona A, Guelar A, Lopez-Colomes JL, Cayla JA. Impact of adherence and highly active antiretroviral therapy on survival in HIV-infected patients. J Acquir Immune Defic Syndr 2002; 30:105-10.

32. Valenti WM. Treatment adherence improves outcomes and manages costs. AIDS Read 2001; 11:7780 .

33. Basuyau F, Josset V, Merle V, Czernichow P. Case fatality and health care costs in HIV-infected patients: evolution from 1992 to 2000 at Rouen University Hospital, France. Int J STD AIDS 2004; $15: 679-84$

34. Beck EJ, Mandalia S, Gaudreault M, Brewer C, Zowall H, Gilmore N et al. The cost-effectiveness of highly active antiretroviral therapy, Canada 1991-2001. Aids 2004; 18:2411-8.

35. Anis AH, Guh D, Hogg RS, Wang XH, Yip B, Craib KJ et al. The cost effectiveness of antiretroviral regimens for the treatment of HIV/AIDS. Pharmaco Economics 2000; 18:393-404.

36. Krentz HB, Auld MC, Gill MJ, HIV Economic Study Group. The changing direct costs of medical care for patients with HIV/AIDS, 1995-2001. CMAJ 2003;169:106-10.

37. Wallace MR, Tasker SA, Shinohara YT, Hill HE, Chapman GD, Miller LK. The changing economics of HIV care. AIDS Patient Care STDS 2001;15:25-9.

38. Phair JP, Bozzette SA. The economics of HIV in the HAART era. [citado el 23/08/2005]. Disponible en: http://clinicaloptions.com/hiv/treatment/pe/

39. Sendi P, Palmer AJ, Gafni A, Battegay M. Highly active antiretroviral therapy: pharmacoeconomic issues in the management of HIV infection. Pharmaco Economics 2001; 19:709-13.

40. Supakankunti S, Tsunekawa K, Phetnoi W. Costing of the national access to antiretroviral program for people living with HIV and AIDS in Thailand. Proceedings of the IHEA 5th World Congress; 2005 Jul 10-13; Barcelona, Spain. Barcelona: UPF; 2005. p.217. 